\title{
Exploratory study of urban resilience in the region of Stuttgart based on OpenStreetMap and literature resilience indicators
}

\author{
H. Sauter ${ }^{1}$, D. Feldmeyer ${ }^{1}$, J. Birkmann ${ }^{1}$ \\ ${ }^{1}$ IREUS, Institute of Spatial and Regional Planning, University of Stuttgart, Germany - holger.sauter@ireus.uni-stuttgart.de
}

Commission IV, WG IV/4

KEY WORDS: Urban resilience, Indicators, Machine learning, R, RStudio, PostGIS

\begin{abstract}
:
The overarching nature of building resilience across disciplines and its inherent positive mutual understanding due to the association with the immune system also amongst the non-scientific community, makes it an attractive and increasing popular concept which everybody seems able to grasp its necessity. Hence, there is an exponential increase, even limited down to the key words "urban resilience", in scientific literature over the last decade. Moreover the concept is also taken up by the New Urban Agenda Habitat III, the SDG goals and the IPCC. Hand in hand with this development the definitions and attempts of operationalization are innumerable. Conjoined, there is a lack of validation of resilience measures, including spatio-temporal aspects but also of the single component of it. Moreover, traditional data sources like census or governmental data miss out on certain important facets making empirical validation impossible and lack the spatio-temporal resolution necessary to cover the characteristics of resilience. Hence, this experimental study explores and develops new spatial indicators through machine learning methods applied to OpenStreetMap data to replicate conventional core indicators. In order to cover all spatial attributes indicators for points, lines and areas are deduced and investigated with supervised and unsupervised algorithms.
\end{abstract}

\section{INTRODUCTION}

The current and expected global threats related to climate change have already highly influenced international policy and the scientific community. Long after the terms sustainability, adaptation and vulnerability entered the international development agendas, resilience as a related but distinct concept (Meerow,2017) experienced a renaissance. Though introduced early by Holling in 1973 (Holling 1973), resilience as a concept is still widely discussed and advanced meanwhile in many fields of research. Holling defined resilience as the "measure of persistence of systems and their ability to absorb change and disturbance and still maintain the same relationship between population or state variables". Though this first explanation focused on ecological systems it delivered a system-related perspective which diffused into other research disciplines. The increasing number of $t$ publications (Meerow und Newell 2017) expressing the ongoing popularity of the concept has resulted in a variety of more differentiated and specialized definitions. Urban resilience and climate resilience of cities in a more specific sense and a special focus on climate adaptation and vulnerability are based on the understanding of these spatial units as being Socio-Ecological systems (SES) where the interconnection of human and ecological systems (Folke, 2006) characterizes their state. Feldmeyer et al. (2019) argues that the Climate resilience "of a city" depends on the ability of its sub-systems to anticipate the consequences of extreme weather and climate change, to resist the negative consequences of these events and to recover essential functions after disturbance quickly, as well as to learn from these events and to adapt to the consequences of climate change in the short and medium term, and transform in the long term. The more pronounced these abilities are, the more resilient a city is to the consequences of climate change (Feldmeyer et al. 2019).

Numerous previous scientific contributions have dealt with the development of indicators measuring resilience at different spatial levels and based on different conceptual frameworks. Hence, many already tested measures or indicators for the assessment of resilience are available (Cutter 2016). Federal authorities such as city administrations are in need for such metrics in order to assess their state of urban resilience and evaluate the effects of their policies and programmes. The majority of these indicators is based on federal or institutional data for the different fields of observation, such as official census data or official landuse surveys etc.. Though such data is often easily available to cities and federal administrations, they can be expensive, differ in temporal frequency or are produced in different structure and formats for each federal state, challenging the feasibility of standardized assessment methods.

Web 2.0 based Volunteered Geographical Information (VGI) technologies could overcome these data-driven obstacles and provide dynamic and standardized data sources even for the federal administrations. The OpenStreetMap (OSM) project, which produces spatial information on a global scale since 2004 through an increasing number of contributors, could be an interesting source of data for the purpose of a standardized database fostering the derivation of socio-ecological indicators. Although the contributions and hence the spatial coverage and density of information still varies significantly around the globe, many regions especially in central Europe around populated areas have a high spatial coverage and could already contain enough information for the distillation of osm-indicators that can supersede and surrogate already recognized measures.

Machine learning algorithms have already been applied for such purposes in the field of economics and or, OSM has served in mixed methods for assessment of landuse and ecological indicators. Nevertheless, it is still unclear if the spatial and thematic information of actual osm datasets within densely mapped areas qualify for the replacement of recognized variables for the assessment of resilience on the municipal level. 
Against this background, the research followed a threefold narrative:

- Development of a technically feasible method to extract OSM features for statistical learning

- $\quad$ Testing of Statistical Learning methods to deduce 4 indicators from OSM

- Identification of spatial characteristics that explain four recognized resilience indicators

Following the narrative listed above, we selected four specific resilience measures that can be easily calculated for the municipal level and compared the results of these official indicators with the full Open Street map dataset for the federal district of Stuttgart. The four selected indicators are 1) feeling of belonging, 2) demographic age structure, 3) business tax revenue and 4) unemployment rate are based on existing literature (Cutter 2016, Cutter et al. 2010, MitFLG 2016) and belong to the socioeconomic dimensions of resilience (comp. table 1). The aim was to derive spatial variables deduced from the point, line and area geometries through their associated tag-information of the OSM data. Tags are plain text key-value pairs (e.g.amenity=restaurant) which contain the information about the attributes and function of objects. The federal district of Stuttgart qualifies well as study area for our research, as it has an already high OpenStreetMap coverage and contribution activity (Arsanjani et al. 2015). The economically strong district is one out of four within the federal state of Baden-Wuerttemberg in Southern Germany with more than 4 million inhabitants, a high density of infrastructure and services and consists of 343 municipalities.

\section{METHODOLOGY}

The methodology consist of four logical evolving parts. Firstly accessing the data. Secondly preprocessing the data. Thirdly, the statistical methods applied. Fourthly, the urban resilience components as response variables for the OSM predictors.

\subsection{Accessing data}

Until now, many helpful tools, websites and API's offering dynamic query abilities and other analytic services regarding the OpenStreetMap database exist. Among them are the famous Overpass-api with the Overpass Turbo online query form, osmstats and osm taginfo, to mention only a few. A very interesting website and API recently developed by HeiGIT (University of Heidelberg) offering the possibility to filter osm data worldwide with the calculation of statistics and counts for specific tags and geometries. The aforementioned API's and services can be efficiently used for the analysis of selected datasets with a limited spatial coverage but they can get slow or just refuse the response when a query exceeds a certain memory threshold. As the scope of our analysis was to reveal the relevant tags and/or keys of importance regarding our research questions, we decided to use full static osmdata because our explorative analysis is based on all geometries and their attribute information of Open street map in the district of Stuttgart.

\subsection{Data preprocessing}

In a first step, the latest full OSM dataset for the district of Stuttgart was downloaded from the Geofabrik website as .pbf file and imported into a PostgreSQL database with activated POSTGIS extension, using the command line program osm2pgsql. We used the default import "style" provided by XY creating POSTGIS layers that contain more than 60 of the most common keys as columns and respective values as rows in the attribute tables of the point, line and polygon geometries. The second step of preprocessing involved the spatial intersection of the osm-geodata with the administrative boundaries of all municipalities within the district of Stuttgart. This enabled us to count unique key-value-pairs with their corresponding sums of area and length for polygon and line geometries for each municipality. Keys and value pairs with little occurrence and spatial relevance where deleted from the outputs. For the spatial intersection of the OSM data with the boundaries of the municipalities (source: http://www.geodatenzentrum.de/) we used QGIS 3.6.

\subsection{Statistical methodology}

The data processing methodology delivered the table with the predictors for modelling the response variables, in our case the four resilience components. The statistical part was done using $\mathrm{R}$ with R Studio (RStudio Team 2018, R Core Team 2019).

2.3.1 Deducing indicators: The first step was to reshape the three tables of points, lines and areas. Each tag consisting of key and value became a single column, where each municipality was a single row. For points the count per municipality, for lines the length in meters and for areas the square meters were the values of the columns. Afterwards all values were divided by the population of the municipality and min max normalized. Now the single joined table had 343 rows of all municipalities and 441 columns named by tag and a value between zero and one.

This extensive list further went through a three step statistically refining and dimension reduction process using the caret package in $\mathrm{R}$ (Kuhn et al. 2017). Firstly, columns where deleted having more than $50 \%$ missing values. Secondly, zero and near zero variance predictors were also removed. Thirdly, highly correlated variables were detected and removed. These three steps reduced the table to 344 predictors.

2.3.2 Regression Tree: The flexible approach of regression trees was selected in order to not make any assumptions about the relationship of the predictors and the response variable.

Regression trees have the benefit of not assuming a linear relation like linear regression. Moreover, the tree visualisation simplify the interpretation and also communication of the results. On the downside regression trees have the problem of over-fitting and hence being very non-robust to small changes in the data (James et al. 2013). Analysing the the data to get a first understanding of them a simple regression tree was calculated with the tree () function of the tree package (Ripley 2014)

2.3.3 Pruned Tree: For the improving the results and increase robustness, but moreover to help determining the major and core predictors the regression tree was pruned in a second step. Which reduces the notes and cut the tree basically down to the core, for balancing better the variance-bias trade off.

2.3.4 Random Forest: Random forest is an advancement of the classic tree due to the nowadays available computational processing power. Instead of one tree thousand trees are modelled. Moreover each time a tree is build also by bootstrapping a training sample is taken. To ensure that not only one very strong predictor is dominating the entire forest and all trees look alike the also a random subset of predictors is taken. Within the R environment the randomForest() function by Liaw and Wiener (2002) is applied. 


\subsection{Resilience indicators}

Four respond variables covering different dimensions were selected (Table 1). Addressing different capacities of urban resilience the possibilities of osm based indicators for estimating them could be tested.

\begin{tabular}{|c|c|c|}
\hline Dimension & Indicator/Response & Source \\
\hline $\begin{array}{l}\text { Community } \\
\text { connectivity }\end{array}$ & Feeling of belonging & Cutter 2016 \\
\hline Social capital & $\begin{array}{l}\text { Demographic age } \\
\text { structure }\end{array}$ & $\begin{array}{l}\text { Cutter et al. } \\
2010\end{array}$ \\
\hline $\begin{array}{l}\text { Economic } \\
\text { strength }\end{array}$ & Business tax revenue & $\begin{array}{l}\text { Feldmeyer et } \\
\text { al. } 2019\end{array}$ \\
\hline Spatial justice & Unemployment & $\begin{array}{l}\text { MitFLG } \\
2016\end{array}$ \\
\hline
\end{tabular}

Table 1.Urban resilience response variables

\section{RESULTS}

The result chapter is split into four parts: First, community connectivity; second, social capital; third, economic strength and fourth, social justice. For each of these four core topics a pruned regression tree and a random forest model will be introduced. The random forest model output is mapped and compared to the response variable.

\subsection{Community connectivity}

Community connectivity plays a key role opposing uncertain threats. Despite its importance, measuring it quantitatively remains unsolved and unsatisfactory. Hence we will use the migration balance of each municipality as a proxy for connectivity and take it as the response variable of the models in order to find spatial attributes related to its patterns.

3.1.1 Regression Tree: Figure 1 shows the regression tree taking the migration balance as response and OSM as predictors. The tag administrative boundary divides the tree on the top first level.

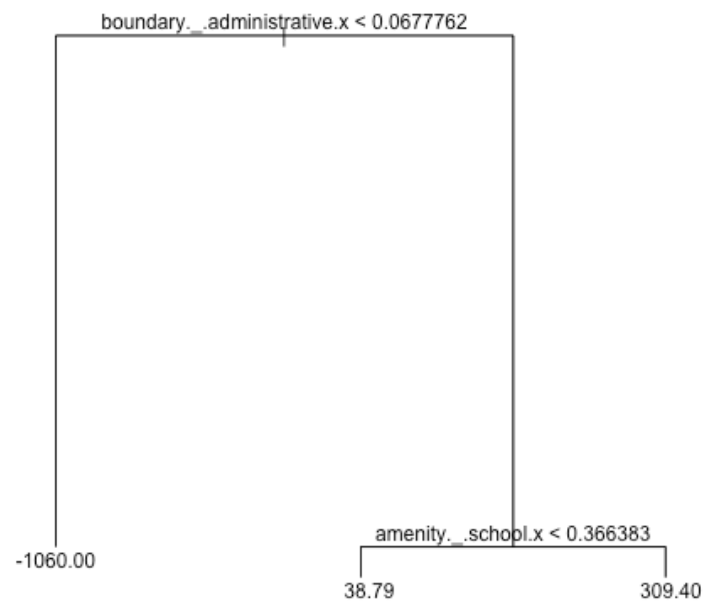

Figure 1. Regression tree feeling of belonging to community

The tree has only one further node with the tag amenity school. In this case over-fitting is no problem and pruning the tree for better robustness is not necessary.
3.1.2 Random Forest: The random forest results are shown in Table 2. The first obvious difference is the top node. This contrast results from the inherent complexities of the 2 models. The regression tree is based only on one random sample of training data and a sample of test data which in our case produced the is dominant boundary administrative tag only followed by the tag amenity school (comp. Figure 1).The in depth analysis with Random forest iterating over thousands of trees generated reveals that the tag barrier lift gate has the highest X.IncMSE over all analysed trees. Except the second tag access private. the remaining tags have a X.IncMSE around two.

\begin{tabular}{|c|c|c|}
\hline $\operatorname{tag}$ & MSE & Purity \\
\hline barrier._.lift_gate & 4,56 & 39737,3 \\
\hline access._.private. $x$ & 3,84 & 26347,7 \\
\hline network._.VVS & 2,80 & 15225,2 \\
\hline covered._.yes.x & 2,51 & 23796,3 \\
\hline power._.generator.x & 2,12 & 49872,7 \\
\hline highway._turning_circle & 2,07 & 114491,9 \\
\hline brand._.Deutsche.Post & 2,06 & 65227,6 \\
\hline shop._.supermarket.y & 2,05 & 3227,2 \\
\hline building._.public & 2,01 & 4429,8 \\
\hline historic._.boundary_stone & 2,00 & 926,5 \\
\hline
\end{tabular}

3.1.3 Mapping feeling of belonging: Assuming that regions with people that are very connected to their region, will show less net out-migration, migration balance is used as an indicator based on statistical data.

Map 1 shows the migration pattern within the region of Stuttgart. For each municipality the net migration is calculated by subtracting the number of people moving out of the municipality from the numbers moving into. Comparing the municipalities each is classified from very low to very high by their quantile.

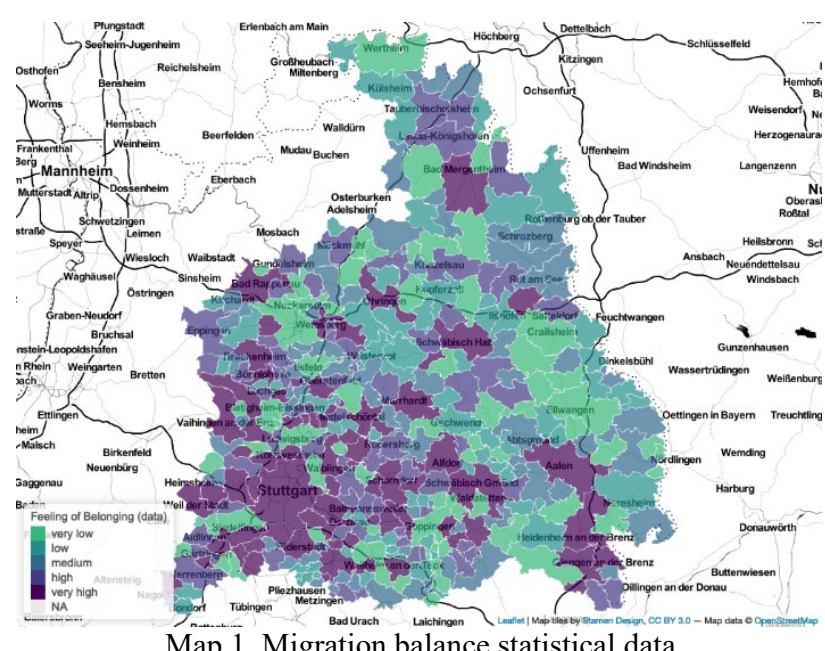

The central region around the city shows the highest plus in migration. The light green parts are at the other end of the scale and mainly located in the Easter part of the region. 


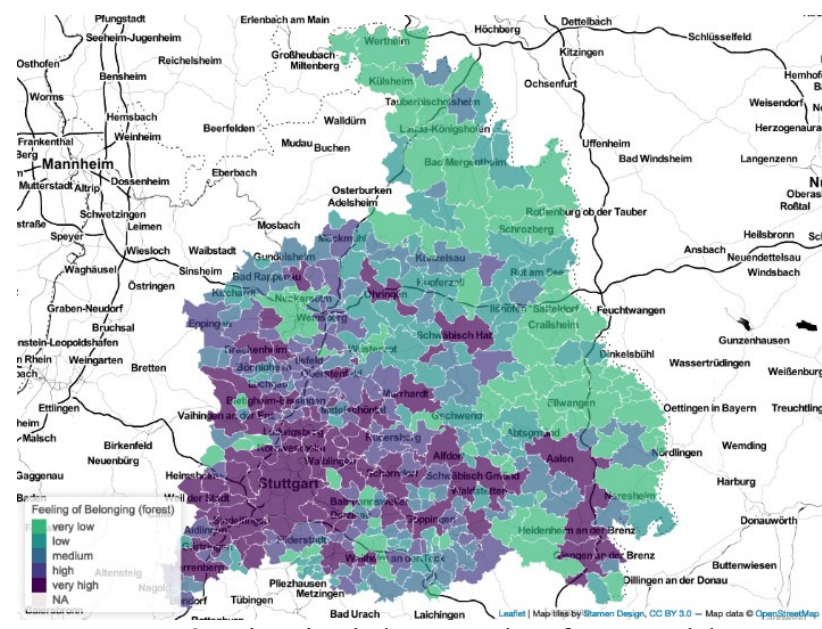

Map 2. Migration balance random forest model

Map 2 is based on the random forest model and its top ten nodes shown in Table 2. The dominant pattern that the central part of the region around Stuttgart has a very high balance, is clearly matched. The highest difference is in the north of the region, where the model does not reflect the positive balance correctly.

\subsection{Social capital}

The population age structure plays into several fields of urban resilience like emergency management but also economy and health. Hence the indicator share of population above 6 and below 65 is taken as response.

3.2.1 Regression Tree: The top level split of the regression tree in Figure 2 analysing the relationship of OSM and age is the tag landuse allotment. The tree is split subsequently into two equally deep branches. The left branch is afterwards dominated by the tag bus yes and the right respectively by boundary public transport.

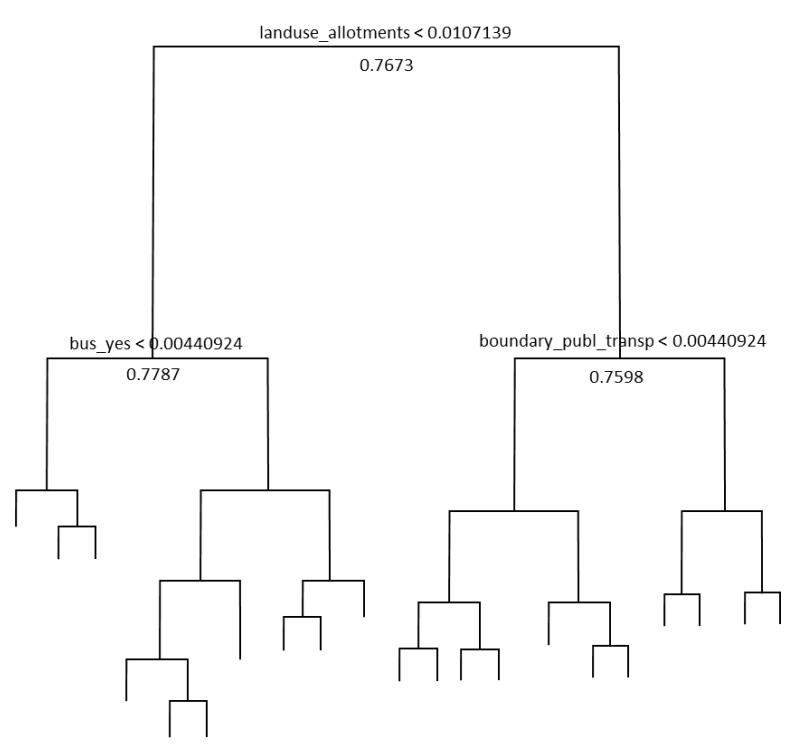

Figure 2. Regression tree age structure (labels of lower branches removed for reasons of readability)

The number of levels, nodes and branches indicates over-fitting by the regression tree.
3.2.2 Pruned Tree: In order to balance the variance-bias trade-off the regression tree is pruned (Figure 3 ). The left branch ends with the bus yes node. The right branch is made by two nodes: boundary public transport and landuse farmland.

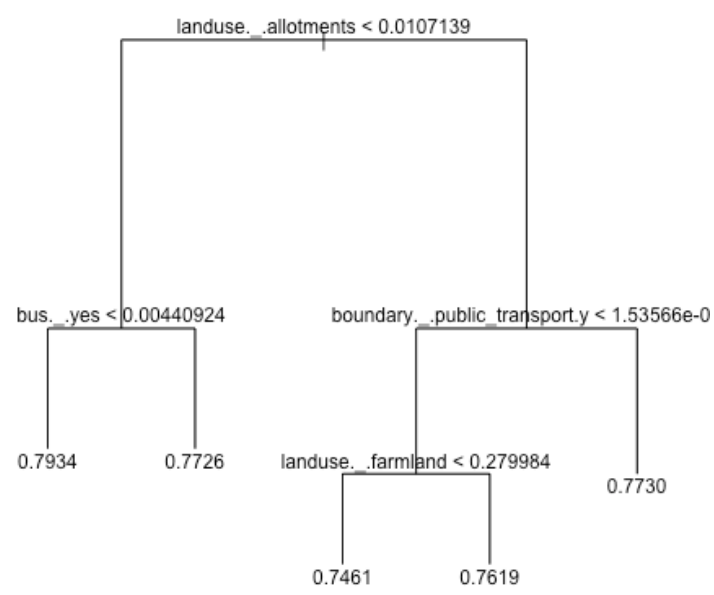

Figure 3. Pruned tree age structure

Consequently the dominant relations of OSM to age are landuse and public transport.

3.2.3 Random Forest: The nodes of the pruned tree are part of the top nodes of random forest (Table 3 )

\begin{tabular}{|c|c|c|}
\hline & MSE & Purity \\
\hline highway._.living_street & 6,63 & 0,0025 \\
\hline landuse._.allotments & 6,37 & 0,0035 \\
\hline boundary._.public_transport & 5,70 & 0,00086 \\
\hline natural._peak & 5,36 & 0,0017 \\
\hline amenity._.pub & 5,01 & 0,0024 \\
\hline seats._.4 & 4,69 & 0,0011 \\
\hline landuse._.farmland & 4,59 & 0,00089 \\
\hline highway._.steps & 4,49 & 0,0021 \\
\hline hiking._yes & 3,76 & 0,0016 \\
\hline operator._.Schwäbischer.Albverein & 3,24 & 0,0010 \\
\hline
\end{tabular}

Only the tag bus yes is not within the top ten nodes ranked by their X.IncMSE.The top node is the tag highway living street, in German called "Spielstraße" which could be loosely translated to "Playground street" which means cars are only allowed to drive a maximum speed of $7 \mathrm{~km} / \mathrm{h}$. The second tag is landuse allotment with also a IncMSE of 6 . Below three tags with an IncMSE of five follow: boundary, public transport, natural peak and amenity pub. The bottom is made by benches to sit down (seats_4), farmland, flights of steps on footways (highway_steps) and recreational hiking possibilities (hiking_yes, operator_Schwäbischer_Albverein).

3.2.4 Mapping share of above 6 under 65: Map 3 shows the indicator share of inhabitants per municipality above the age of 6 and below 65 years. There is an obvious belt north of the city of Stuttgart from West to East with a higher share. 


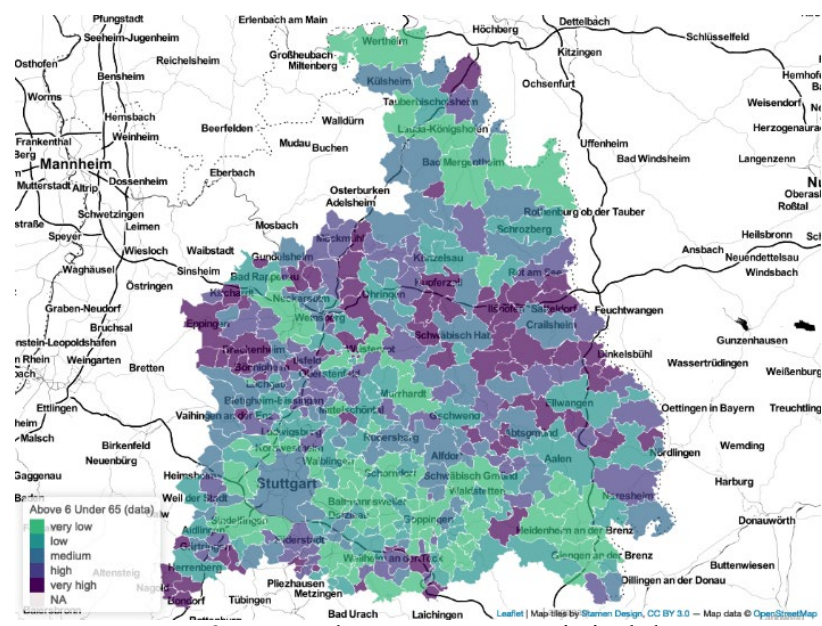

Map 3. Mapped age structure statistical data

Map 4 is based on the values of the random forest model. Compared to the statistical data the dominant patterns remain. The model in general is doing well in reproducing major trends but fails to detect abnormalities. The city of Stuttgart and its surroundings is homogeneous OSM wise but the statistical data show a lower proportion of very young and older people in the city itself.

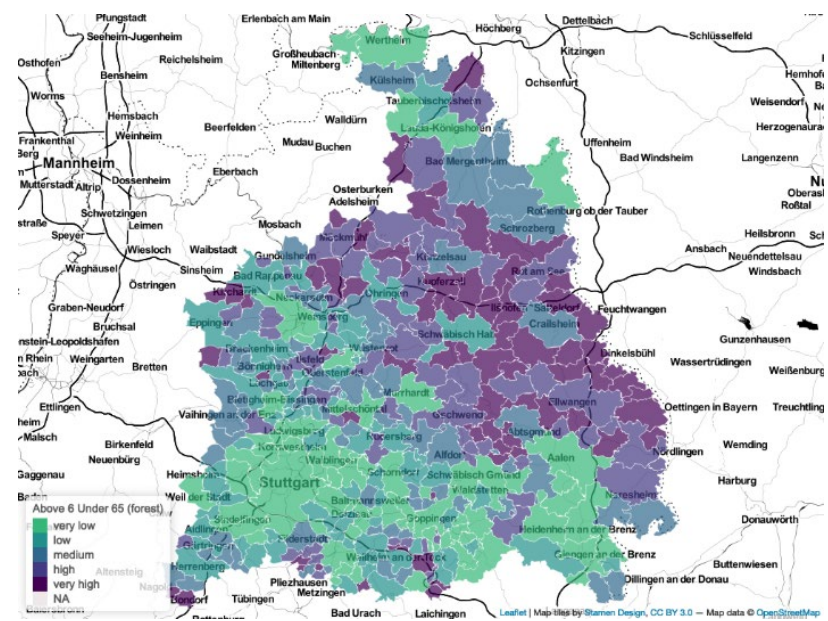

Map 4. Mapped age structure random forest model

\subsection{Economic strength}

The economic strength is measured by business tax per capita and municipality within the district of Stuttgart. As an important income source for the local government it reflects also the financial resources to cope in the short term with negative impacts and the ability to implement long term protection and climate change adaptation measures.

3.3.1 Regression Tree: The first decision is made by the tag surface paved (Figure 4). On the second level the left branch is further divided by building industrial and the right branch by landuse orchard, which is the last node of the right branch. The left branch further is defined by: leisure stadium, landuse commercial, landuse farmyard, man-made silo and barrier fencey.

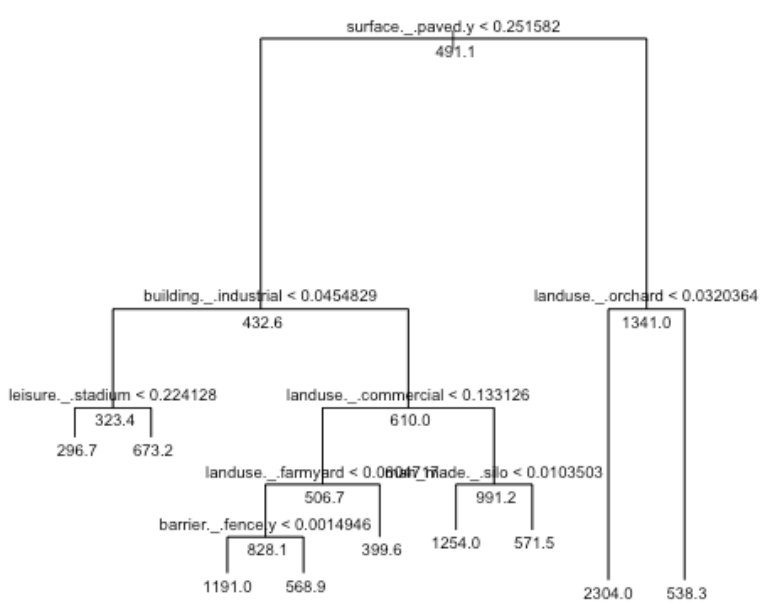

Figure 4. Regression tree business tax per capita

3.3.2 Pruned Tree: The last levels of the regression tree are cut by the pruning algorithm. The main selected predictors for the pruned tree are: surface paved, building industrial, landuse orchard and landuse commercial.

Figure 5. Pruned tree bushiness tax per capita

3.3.3 Random Forest: Only landuse orchard of the pruned tree nodes is not in the top ten of the random forest (Table 4). Quite intuitive building industrial, landuse commercial, surface

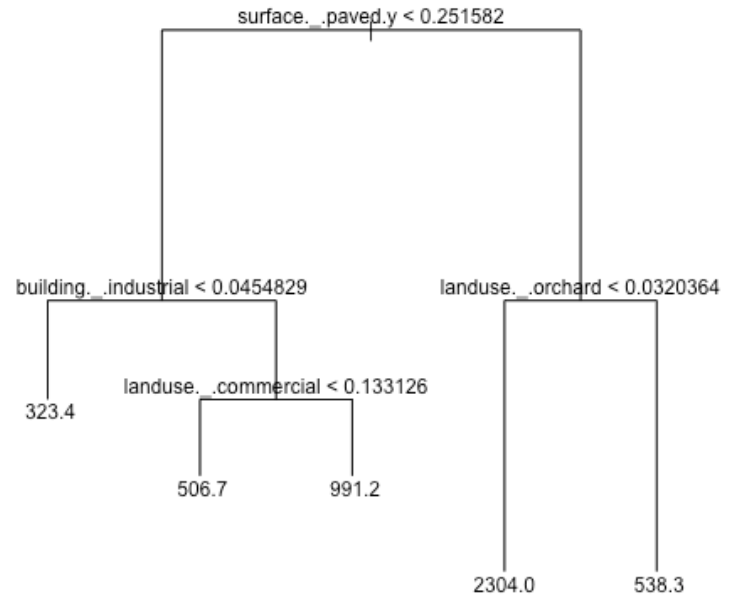

paved and landuse industrial are strong predictors for business tax.

\begin{tabular}{|c|c|c|}
\hline & MSE & Purity \\
\hline building._.industrial & 5,61 & 993657,8 \\
\hline access._.private.y & 4,09 & 1934836,1 \\
\hline landuse._.commercial & 4,06 & 941009,9 \\
\hline landuse._.industrial & 3,10 & 664165,8 \\
\hline oneway._no & 2,82 & 119253,9 \\
\hline surface._.paved.y & 2,47 & 1760668,2 \\
\hline railway._.level_crossing & 2,29 & 43137,6 \\
\hline
\end{tabular}




\begin{tabular}{lll} 
area._yes & 2,29 & 52416,0 \\
denomination._new_apostolic & 2,14 & 62933,1 \\
entrance._yes & 2,09 & 152156,6 \\
\cline { 1 - 2 } Table 4. Random forest & age structure
\end{tabular}

3.3.4 Mapping tax revenue: The business tax in the region of Stuttgart has a strong cluster around the city of Stuttgart (Map 5). Other important tax revenues are generated in the East of the district, although discontinuously mixed with the lowest category.

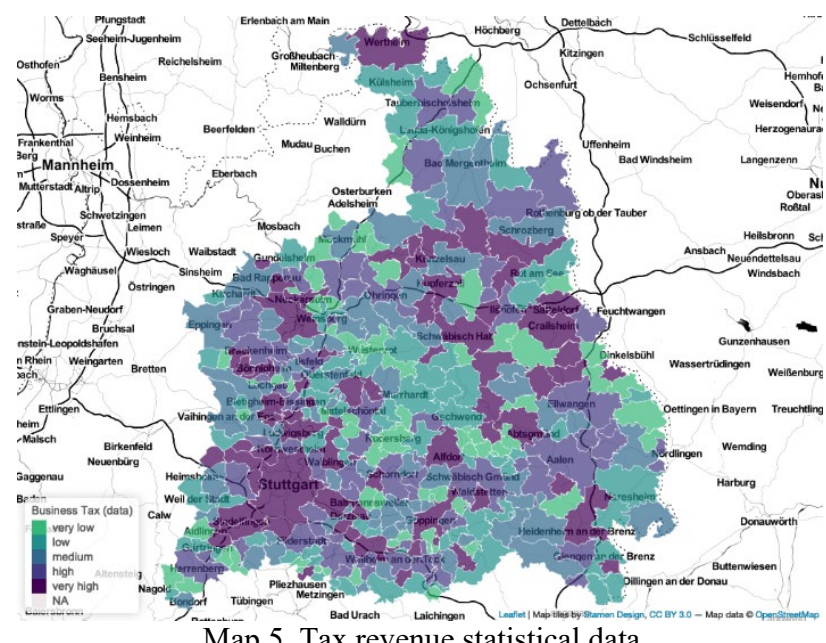

The random forest models overestimate the larger surrounding areas of the city of Stuttgart and its neighbours (Map 6). Similar to the previous maps the North-East are of Stuttgart is underestimated by the model.

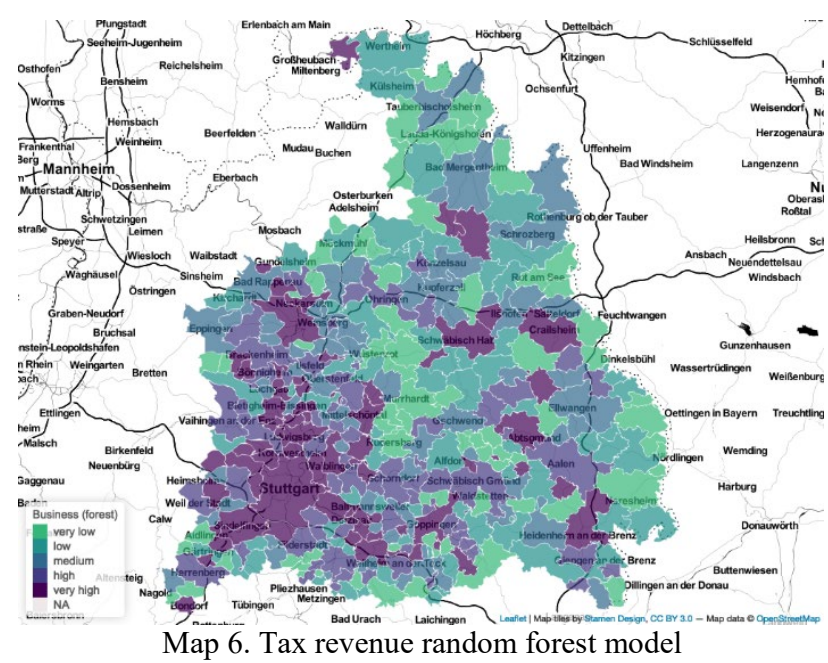

\subsection{Spatial justice}

The fourth topic of analysis is spatial justice. With the proxy of unemployment the spatial justice of the system can be evaluated. Assuming that unemployed people are more dependent on the services provided in their closer proximity than the working population (e.g. commuters), their spatial distribution of this indicator, reveals possible marginalisation if they are pushed out of regions with a high service density.
3.4.1 Regression Tree: the regression tree is dominated by power substation (Figure 6). On the second level boundary public transport and boundary administrative divide the left respectively the right branch. The left branch further splits into six levels showing overfitting. The right branch is smaller and divides only three deeper levels.

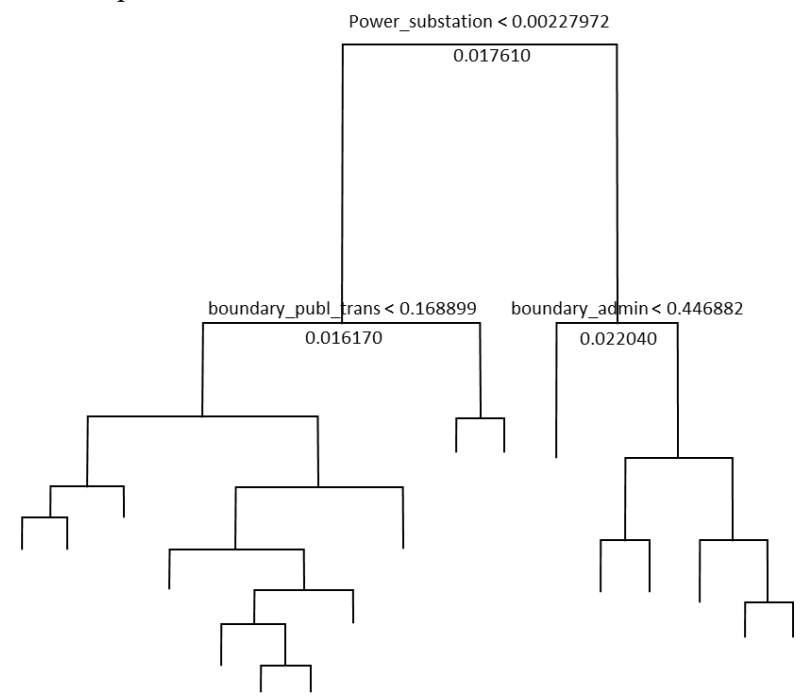

Figure 6. Unemployment regression tree labels of lower branches removed for reasons of readability)

3.4.2 Pruned Tree: the left brunch is trimmed to only one further node, boundary public transport (Figure 7). The right branch previously the shorter one now remains longer and is defined by the predictors consecutively; boundary administrative, place town and operator Deutsche Telekom.

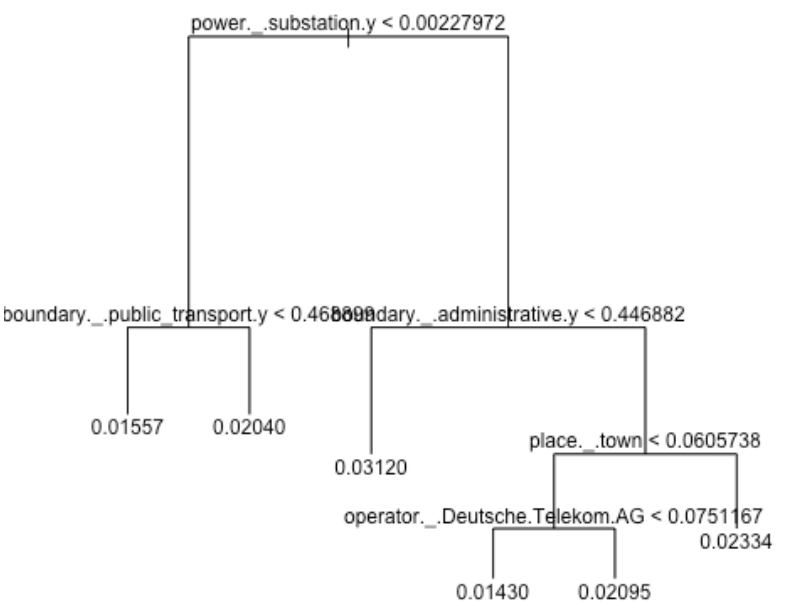

Figure 7. Pruned tree unemployment

3.4.3 Random Forest: The random forest models further explains the underlying data structure and pictures a slightly different picture. Within the top ten nodes the pruned tree nodes are not part (Table 5). Highest ranked according to the x.Inc.MSE is power substation. Followed by highway pedestrian. The third one is leisure sports centre which is ranked closely to the fourth landuse orchard. Further down amenity townhall, leisure stadium, area yes, railway station, building comerical and barrier hedge complete the top ten.

\begin{tabular}{lll} 
& MSE & Purity \\
\hline power._.substation.y & 6,08 & 0,00021198 \\
\cline { 1 - 1 } highway._.pedestrian.x & 5,54 & 0,00021274 \\
\hline
\end{tabular}




\begin{tabular}{|c|c|c|}
\hline leisure._.sports_centre.x & 4,34 & $9,80 \mathrm{E}-05$ \\
\hline landuse._.orchard & 4,21 & $6,18 \mathrm{E}-05$ \\
\hline amenity._.townhall.y & 3,87 & $5,62 \mathrm{E}-05$ \\
\hline leisure._.stadium & 3,68 & $6,81 \mathrm{E}-05$ \\
\hline area._yes & 3,25 & 0,00019855 \\
\hline railway._.station & 3,23 & $4,61 \mathrm{E}-05$ \\
\hline building._.commercial & 3,04 & $1,94 \mathrm{E}-05$ \\
\hline barrier._.hedge & 2,94 & $2,82 \mathrm{E}-05$ \\
\hline
\end{tabular}

3.4.4 Mapping unemployment: in general the unemployment of the region of Stuttgart is very low. To still visualise differences the quantile method ranks the municipalities within the region into five classes. The Western and Northern part shows the influence of smaller centres and more inhomogeneous pattern.

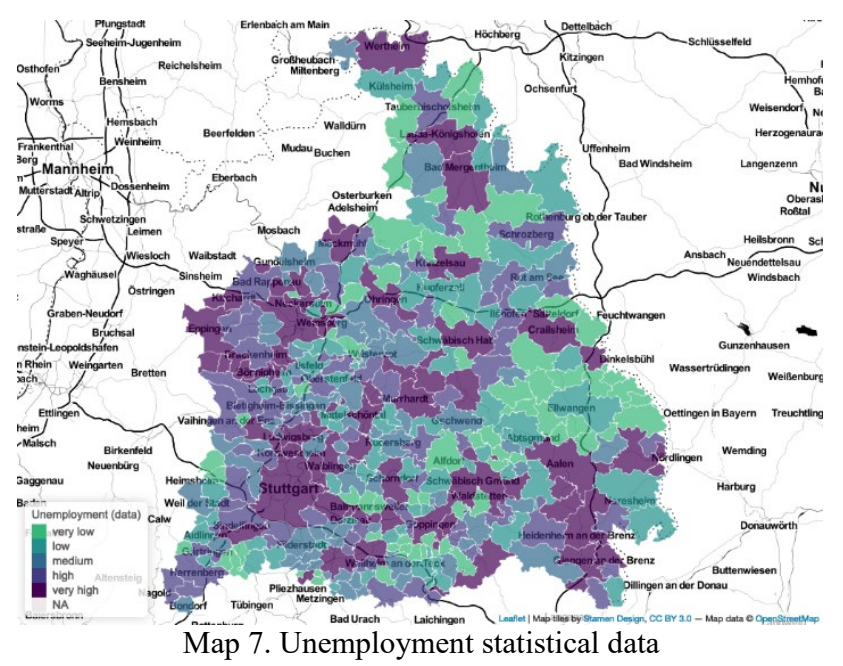

Map 8 is in comparison the result of the random forest model on unemployment. It shows a high congruency matching the major trends as well as small patterns in the Easter and Northern part.

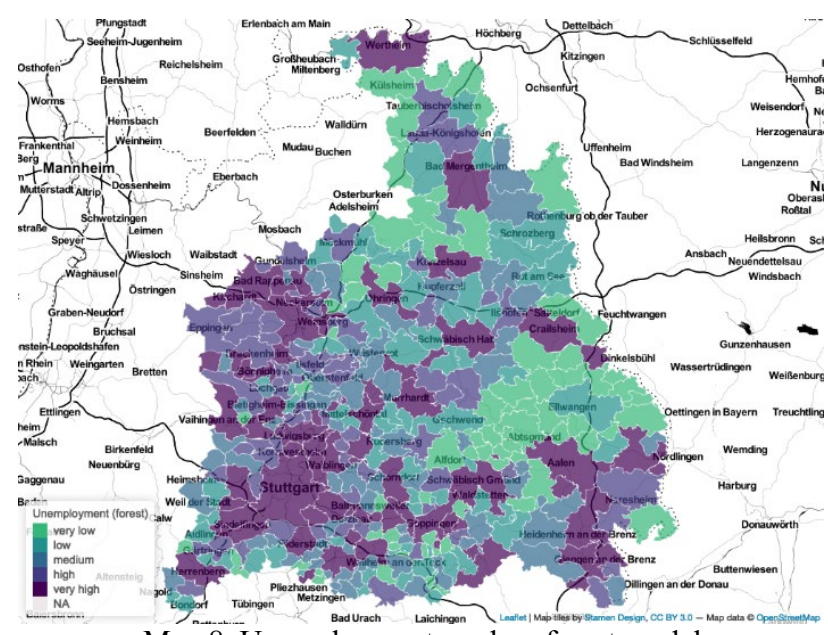

Map 8. Unemployment random forest model

\section{DISCUSSION}

The exploratory approach deducing socio-economic indicators from OSM yielded manifold insights into a not yet explored opportunity of volunteered geographic information (VGI).

Firstly, the access via API was challenging and the amount of data exceeded the limitations of the API. Hence, it was necessary to set up a local PostGIS database for indicator development and data handling. Which again challenged the limitation of standard hardware. Nevertheless, a good standard workstation was able to rifle through the data and structure it. Secondly, keys and tags and their spellings and ordering increased the number of indicators but also decreased the spatial coverage of each of them. Which resulted in a loss of information due to statistical reasons as indicators with a high amount of missing values were fully excluded. Thirdly, this paper focused on the first part of indicator development. From data handling, structuring and exploratory modeling and limitations and challenges with standard hardware. Consequently accuracy, robustness and transferability of models to other regions remain only partially touched. Fourthly, for an analysis of more subjective or qualitative attributes of urban resilience, such as the "feeling of belonging", the response for supervised statistical learning is only very limited. Here, mixed methods approaches including surveys, interviews and stakeholder network analysis might be necessary to quantitatively access more qualitative attributes of urban resilience. Fifthly, the entirely explorative and data driven approach provided surprising insights into relations of phenomenons. Moreover, it reduces bias induced through key and tag selection. On the other hand it is hard to determine true cause effect relations which limits the exploratory power of the models and reduces meaning in the sense of providing clear recommendations for action.

Still having said this, the opportunities for adding important missing pieces to urban resilience which are not yet uncovered outweigh its inherent challenges.

\section{CONCLUSION}

The present research contributes to the understanding of urban resilience by exploring spatial attributes related to its components.

Municipalities are built by a complex interaction of spatial services and attributes. OSM provides a unique interdisciplinary and cross cutting database. Spatial indicators deduced from points, lines and areas are able to mirror socio-economic characteristics. Important capacities and assets of urban resilience are engraved into OSM. The presented work-flow and methodology of statistical learning with $\mathrm{R}$ and POSTGIS provides the necessary environment of exploring its potential with limited computational resources.

It remains challenging to quantify important social characteristics of urban resilience as well as institutional resilience. Hence further research including mixed methods might foster resilience and help decision makers to make informed decisions. 


\section{REFERENCES}

Arsanjani, J. J., Mooney, P.; Helbich, M.; Zipf, A., 2015. An exploration of future patterns of the contributions to OpenStreetMap and development of a Contribution Index. Transactions in GIS 19 (6), 896-914.

Cutter, S. L., Burton, C. G., Emrich, C. T., 2010. Disaster resilience indicators for benchmarking baseline conditions. Journal of Homeland Security and Emergency Management.

Cutter, S. L.,2016. The landscape of disaster resilience indicators in the USA. Natural Hazards: Journal of the International Society for the Prevention and Mitigation of Natural Hazards, 80, 2, 741758 .

Feldmeyer, D., Wilden, D., Kind, C., Kaiser, T., Goldschmidt, R., Diller, C., Birkmann, J.,2019. Indicators for Monitoring Urban Climate Change Resilience and Adaptation. Sustainability 11,2931

Folke, C., 2006. Resilience. The emergence of a perspective for social-ecological systems analyses. Global Environmental Change 16 (3), 253-267. DOI 10.1016/j.gloenvcha.2006.04.002.

Holling, C. S., 1973. Resilience and Stability of Ecological Systems. Annual Review of Ecology and Systematics 4 (1), 123. DOI: 10.1146/annurev.es.04.110173.000245.

James, G., Witten, D., Hastie, T., Tibshirani, R., 2013. An introduction to statistical learning. Corrected at 8th printing 2017. New York: springer.

Liaw A., Wiener, M., 2002. Classification and Regression by randomForest. R News 2(3), 18--22.

Meerow, S., Newell, J. P., 2017. Urban resilience for whom, what, when, where, and why? Urban Geography 11 (1), 1-21. DOI: 10.1080/02723638.2016.1206395

Kuhn, M., Contributions from Jed Wing, Steve Weston, Andre Williams, Chris Keefer, Allan Engelhardt, Tony Cooper, Zachary Mayer, Brenton Kenkel, the R Core Team, Michael Benesty, Reynald Lescarbeau, Andrew Ziem, Luca Scrucca, Yuan Tang, Can Candan and Tyler Hunt. (2017). caret: Classification and Regression Training. R package version 6.0-78. https://CRAN.Rproject.org $/$ package $=$ caret

Mitigation Framework Leadership Group (MitFLG). Mitigation Framework Leadership Group Draft Concept Paper. Draft Interagency Concept for Community Resilience Indicators: Washington, DC, USA, 2016.

OpenStreetMap contributors, 2019. stuttgart-regbez-latest.osm [Data file from 2019-04-16]. Retrieved from https://download.geofabrik.de

QGIS Development Team, 2019. QGIS Geographic Information System. Open Source Geospatial Foundation Project. http://qgis.osgeo.org

The PostgreSQL Global Development Group, 2019. PostgreSQL (release 10.8). https://www.postgresql.org/
R Core Team, 2019. R: A language and environment for statistical computing. R Foundation for Statistical Computing, Vienna, Austria. URL https://www.R-project.org/.

Ripley, B., 2014. Package "tree". Retrieved from http://cran.rproject.org/web/packages/tree/index.html

RStudio Team, 2018. RStudio: Integrated Development for R. RStudio, Inc., Boston, MA URL http://www.rstudio.com/. 